\section{BEM-ESTAR DOS PROFESSORES DE EDUCAÇÃO FÍSICA ESCOLAR: UMA REVISÃO DE LITERATURA}

\section{WELL-BEING OF SCHOOL PHYSICAL EDUCATION TEACHERS: A LITERATURE REVIEW}

Almir Fagundes Neves Neto ${ }^{1}$ Cláudio Bispo de Almeida ${ }^{1, *}$ / Rodrigo Araújo Pereira ${ }^{1}$ / Jorge Both ${ }^{2}$

\section{INTRODUÇÃO}

Percebe-se que a educação é um processo em constante transformação, mesmo sendo a docência uma das profissões mais antigas da humanidade (LEMOS; CRUZ, 2005). A prática docente é passível de fazer com que docentes possam desenvolver distúrbios de saúde por fatores externos, atribuições diárias, má alimentação, má qualidade no sono, ocasionando uma má qualidade de vida e estresse (MELEIRO, 2002), que podem afetar, assim, a saúde física e mental dos professores (ESTEVE, 1999).

Para Reis et al. (2005) a profissão docente é uma das mais expostas a ambientes conflituosos e de alta exigência de trabalho. Assim, diversos fatores estressores tendem a afetar os profissionais dessa área, como salas de aula superlotadas, má remuneração, excesso de carga horária, falta de materiais didáticos, dentre tantos outros fatores que contribuem para a insatisfação dos profissionais ao exercerem suas atividades.

O docente sofre uma "crise de identidade" pelos fatores a que são expostos e o estresse frequente (TEODORO, 1994). É inegável que a violência contra professores, brigas administrativas, baixos salários, dentre outras exposições, justifiquem a necessidade urgente da mudança de visão da comunidade acerca dos profissionais docentes para modificar esse quadro desagradável (BOTH, 2008).

\section{RESUMO}

O objetivo do presente estudo foi observar como o bemestar dos professores de educação física da rede escolar brasileira vem sendo abordado na produção acadêmica do período de 2000 a 2010 . Trata-se de um estudo de revisão de literatura, realizado no site de busca google acadêmico, utilizando-se as seguintes combinações: qualidade de vida and docência or professor and educação física. Os estudos se caracterizaram como de metodologia quantitativa, e os resultados revelaram que: o nível de satisfação aumentou com o passar dos anos na profissão; as condições de saúde não favoráveis acabam afetando a saúde dos docentes; destacaram a importância da contribuição do estilo de vida e da qualidade de vida para o trabalhador docente, sendo diretamente relacionado ao bem-estar. Assim, os estudos convergem no sentido de que, para melhorar o bem-estar dos professores de educação física, deve haver um investimento governamental na educação, proporcionando melhores condições de trabalho e incentivo profissional.

Palavras-chave: Promoção da saúde. Docência. Educação Física.

\section{ABSTRACT}

The objective of the present study was to observe how the well-being of physical education teachers in the Brazilian school system has been addressed in academic production from 2000 to 2010. This is a literature review study, conducted in the google academic search site, using the following combinations: quality of life and teaching or teacher and physical education. The studies were characterized as a quantitative methodology, and the results revealed that: the level of satisfaction increased with the passing of the years in the profession; the unfavorable health conditions end up affecting the health of the teachers; highlighted the importance of the contribution of lifestyle and quality of life for the teaching worker, being directly related to well-being. Thus, the studies converge in the sense that, to improve the well-being of physical education teachers, there must be a government investment in education, providing better working conditions and professional incentives.

Keywords: Health promotion Teaching. Physical Education.

Submetido em: 14 de jun. 2018

Aceito em: 14 de abr. 2020

${ }^{1}$ Universidade do Estado da Bahia - UNEB, Guanambi, Bahia - Brasil.

${ }^{2}$ Universidade Estadual de Londrina, Londrina, Paraná - Brasil.

*E-mail para correspondência: cbalmeida@uneb.br 
O bem-estar do trabalhador docente sofre a influência dos principais indicadores que podem ser alterados e que afetam diretamente a saúde do profissional de educação, ou seja, a percepção sobre o trabalho e os comportamentos do estilo de vida (BOTH, 2011). Destaca-se que o professor de educação física sofre um desgaste maior em relação aos outros docentes, porque passa a maior parte do tempo da aula em pé, na maioria das vezes exposto ao sol, além de elevar o tom da voz, prejudicando suas cordas vocais, situações que contribuem para a diminuição da sensação de bem-estar. Por isso, não se pode desconsiderar os efeitos de aspectos que englobam o domínio meio ambiente (SILVA; NUNES, 2009). Assim, tendo em vista as condições de trabalho e sua relação com a saúde, torna-se importante observar como o bem-estar dos professores de educação física da rede escolar brasileira vem sendo abordado na produção acadêmica do período de 2000 a 2010.

\section{MATERIAL E MÉTODOS}

Trata-se de uma revisão de literatura, de caráter qualitativo. Foram incluídos na pesquisa produções em português, publicadas no período de 2000 a 2010, que estudaram o bemestar dos professores de educação física do ensino básico, e que estavam com texto completo disponível. A pesquisa foi realizada no sítio de busca Google Acadêmico. Foi utilizada a seguinte combinação de palavras chaves e indicadores booleanos: qualidade de vida and docência or Professor and educação física.

Após a busca, uma análise inicial foi realizada por meio da seleção com base nos títulos dos manuscritos. Em seguida, fez-se uma análise dos resumos, das publicações selecionadas (50 manuscritos) obtidos e lidos na íntegra e, examinados de acordo com o objetivo do presente estudo. As realizações das leituras consistiram em quatro etapas: a leitura exploratória, a qual objetivou distinguir quais textos foram relevantes para a pesquisa; a leitura seletiva, feita no intuito de verificar se o material selecionado correspondia aos temas bem-estar ou qualidade de vida e docência; a leitura analítica, que consistiu na busca para a resposta do problema; e por fim, a leitura interpretativa, que relacionou os textos selecionados com o objetivo da pesquisa (GIL, 2006).

De acordo com Gil (2006), a análise tem como objetivo organizar os dados de forma tal que possibilite o fornecimento de resposta ao problema proposto pela investigação. A forma de análise utilizada pela presente pesquisa é a análise de conteúdo, que visou obter, por procedimentos sistemáticos, os objetivos das mensagens e indicadores que permitiam a inferência de conhecimentos relativos às condições de produção.

\section{RESULTADOS E DISCUSSÃO}

Utilizou-se o sítio eletrônico google acadêmico, no qual foram disponibilizados 16.126 com a combinação proposta na busca, dos quais doze foram utilizados na pesquisa por atenderem aos critérios de inclusão e exclusão pré-estabelecidos. Observou-se que a maior parte das publicações encontradas: foram publicadas no ano de 2008 (BOTH, 2008; BOTH; NASCIMENTO; BORGATTO, 2008a; BOTH; NASCIMENTO; BORGATTO, 2008b; FARIAS et al., 2008) e são estudos quantitativos (BOTH; NASCIMENTO; BORGATTO, 2008a; BOTH; NASCIMENTO; BORGAT-
TO, 2008b; FARIAS et al., 2008; FEITOSA; RODRIGUES, 2010; LEMOS, 2007; LEMOS; NASCIMENTO; BORGATTO, 2007; MOREIRA et al., 2010a; MOREIRA et al., 2010b; SILVA; NUNEZ, 2009) (Quadro 1).

Os estudos avaliados (Quadro 1) abordaram os seguintes temas de investigação: doenças e condições de saúde relacionadas ao exercício da docência (BOTH; NASCIMENTO; BORGATTO, 2008a; MOREIRA et al., 2010b; SANTINI, 2004; SILVA; NUNEZ, 2009); condições e satisfação no trabalho do professor (BOTH, 2008; FARIAS et al., 2008; GASPARINE et al., 2006; LEMOS; NASCIMENTO; BORGATTO, 2007; MOREIRA et al., 2010a; MOREIRA et al., 2010b); estilo de vida e práticas que melhorem o bem-estar do trabalhador docente (BOTH; NASCIMENTO; BORGATTO, 2008b; FEITOSA; RODRIGUES, 2010). Tais estudos evidenciaram preocupação com o bemestar dos docentes de educação física, tendo em vista as abordagens envolvem, além da preocupação com a saúde, a qualidade de vida e o estilo de vida.

\section{DOENÇAS E CONDIÇÕES DE SAÚDE RELACIONADAS AO EXERCÍ́CIO DA DOCÊNCIA}

A profissão docente vem se tornando cada dia menos atraente, devido às condições de trabalho e as relações interpessoais estabelecidas entre professor e aluno e, também, por conta da desvalorização financeira (BORGATTO, 2008a; BOTH; NASCIMENTO; SILVA; NUNEZ, 2009). Além disso, doenças como o estresse e a Síndrome do Esgotamento Profissional (SEP), têm afetado os professores (SANTINI, 2004). Todos esses fatores contribuem para o cerceamento da atuação docen- 
te. Ao se abordar o bem-estar do trabalhador docente, tem que se levar em consideração não somente a ausência ou a presença de doenças, mas, também, a promoção de hábitos que conduzam à boa saúde física e mental, influenciando positivamente a vida do indivíduo (MOREIRA et al., 2010b).

Quadro 1. Estudos publicados sobre o bem-estar de docentes de educação física no período de 2000 a 2010.

\begin{tabular}{|c|c|c|}
\hline Autores & \begin{tabular}{|l|} 
Metodologia \\
utilizada
\end{tabular} & Considerações \\
\hline $\begin{array}{l}\text { Santini } \\
(2004) .\end{array}$ & \begin{tabular}{|l|} 
Pesquisa \\
descritiva, de \\
caráter \\
qualitativo.
\end{tabular} & $\begin{array}{l}\text { O trabalho docente } \\
\text { revelou-se uma prática } \\
\text { profissional marcada } \\
\text { por sentimentos } \\
\text { negativos que com- } \\
\text { prometem a qualidade } \\
\text { do trabalho acumu- } \\
\text { lando com o passar do } \\
\text { tempo, reaçóes físicas, } \\
\text { psíquicas comporta- } \\
\text { mentais e defensivas. }\end{array}$ \\
\hline $\begin{array}{l}\text { Gaspari- } \\
\text { ni et al. } \\
(2006)\end{array}$ & \begin{tabular}{|l|} 
Pesquisa \\
qualitativa. \\
Número de \\
participantes \\
da pesquisa = \\
21 professo- \\
res de \\
educação \\
física dos \\
ensinos \\
fundamental \\
e médio.
\end{tabular} & $\begin{array}{l}\text { Conhecimento prévio } \\
\text { das histórias de vida } \\
\text { das pessoas - suas } \\
\text { práticas, influências } \\
\text { sociais, relações } \\
\text { familiares, formação } \\
\text { religiosa ou classe } \\
\text { econômica e política. }\end{array}$ \\
\hline $\begin{array}{l}\text { Lemos } \\
\text { (2007). }\end{array}$ & \begin{tabular}{|lr|}
\multicolumn{2}{l|}{ Pesquisa } \\
descritivo- \\
exploratória \\
que utilizou \\
como instru- \\
mentos de \\
coleta de \\
dados os \\
questionários \\
QVT-PEF e o \\
Perfil do \\
Estilor de \\
Vida Indivi- \\
dual. \\
Número de \\
participantes \\
da pesquisa = \\
380 professo- \\
res de \\
educação \\
física.
\end{tabular} & $\begin{array}{l}\text { O aumento do nível de } \\
\text { satisfação da qualida- } \\
\text { de de vida no trabalho } \\
\text { está associado ao } \\
\text { avanço na carreira } \\
\text { docente, onde os } \\
\text { professores do ciclo } \\
\text { de estabilização } \\
\text { apresentaram os níveis } \\
\text { mais elevados. }\end{array}$ \\
\hline $\begin{array}{l}\text { Lemos, } \\
\text { Nasci- } \\
\text { mento e } \\
\text { Borgatto } \\
(2007) .\end{array}$ & \begin{tabular}{|l|} 
Estudo \\
quantitativo; \\
Número de \\
participantes \\
da pesquisa = \\
380 professo- \\
res de \\
educação \\
física.
\end{tabular} & $\begin{array}{l}\text { O aumento do nível de } \\
\text { satisfação da qualida- } \\
\text { de de vida do traba- } \\
\text { lhador está associado } \\
\text { ao avanço na carreira } \\
\text { docente. }\end{array}$ \\
\hline $\begin{array}{l}\text { Farias et } \\
\text { al. } \\
(2008) \text {. }\end{array}$ & $\begin{array}{l}\text { Estudo } \\
\text { descritivo } \\
\text { exploratório, }\end{array}$ & $\begin{array}{l}\text { Com o avanço na } \\
\text { carreira, os professo- } \\
\text { res estão mais satisfei- }\end{array}$ \\
\hline
\end{tabular}

\begin{tabular}{|c|c|c|}
\hline & $\begin{array}{l}\text { de caráter } \\
\text { transversal. } \\
\text { Número de } \\
\text { participantes } \\
\text { da pesquisa = } \\
380 \text { professo- } \\
\text { res de } \\
\text { educação } \\
\text { física. } \\
\end{array}$ & $\begin{array}{l}\text { tos com a qualidade } \\
\text { de vida no trabalho. }\end{array}$ \\
\hline $\begin{array}{l}\text { Both } \\
(2008) .\end{array}$ & $\begin{array}{l}\text { Estudo de } \\
\text { caso. }\end{array}$ & $\begin{array}{l}\text { A elevada associação } \\
\text { entre algumas dimen- } \\
\text { sões da Qualidade de } \\
\text { Vida no Trabalho } \\
\text { Docente (QVTD) com } \\
\text { o ciclo de desenvol- } \\
\text { vimento profissional } \\
\text { revelou que, com o } \\
\text { passar dos anos de } \\
\text { docência, os professo- } \\
\text { res tendem a ficar } \\
\text { menos satisfeitos com: } \\
\text { a autonomia no } \\
\text { trabalho, salários e } \\
\text { condições de trabalho, } \\
\text { o que pode ter influ- } \\
\text { enciado na avaliação } \\
\text { do computo geral da } \\
\text { QVTD. }\end{array}$ \\
\hline $\begin{array}{l}\text { Both, } \\
\text { Nasci- } \\
\text { mento e } \\
\text { Borgatto } \\
(2008 \mathrm{a})\end{array}$ & $\begin{array}{l}\text { Investigação } \\
\text { descritiva, de } \\
\text { corte trans- } \\
\text { versal. } \\
\text { Número de } \\
\text { participantes } \\
\text { da pesquisa = } \\
580 \text { docentes. }\end{array}$ & $\begin{array}{l}\text { Apesar da estabilidade } \\
\text { do perfil positivo, ao } \\
\text { longo da carreira } \\
\text { docente, na maioria } \\
\text { das dimensões do } \\
\text { estilo de vida, reco- } \\
\text { menda-se o desenvol- } \\
\text { vimento de ações de } \\
\text { formação aos profes- } \\
\text { sores, para que } \\
\text { possam auxiliar no } \\
\text { controle do estresse } \\
\text { bem como na adoção } \\
\text { de comportamentos } \\
\text { alimentares mais } \\
\text { saudáveis. }\end{array}$ \\
\hline $\begin{array}{l}\text { Both, } \\
\text { Nasci- } \\
\text { mento e } \\
\text { Borgatto } \\
(2008 b)\end{array}$ & $\begin{array}{l}\text { Investigação } \\
\text { descritivo- } \\
\text { exploratória, } \\
\text { de corte } \\
\text { transversal. } \\
\text { Número de } \\
\text { participantes } \\
\text { da pesquisa = } \\
580 \text { docentes. }\end{array}$ & $\begin{array}{l}\text { Apesar da maioria dos } \\
\text { professores catarinen- } \\
\text { ses estarem satisfeitos } \\
\text { com a qualidade de } \\
\text { vida no trabalho os } \\
\text { resultados destacaram } \\
\text { a maior insatisfação } \\
\text { dos docentes das } \\
\text { regiões: Grande } \\
\text { Florianópolis e Norte } \\
\text { Planalto nos compo- } \\
\text { nentes: compensação } \\
\text { salarial, autonomia em } \\
\text { utilizar os conheci- } \\
\text { mentos no trabalho e } \\
\text { relações pessoais no } \\
\text { ambiente de trabalho. }\end{array}$ \\
\hline $\begin{array}{l}\text { Silva e } \\
\text { Nunez } \\
(2009) .\end{array}$ & $\begin{array}{l}\text { Pesquisa } \\
\text { exploratória. } \\
\text { Número de } \\
\text { participantes } \\
\text { da pesquisa = } \\
69 \text { professo- } \\
\text { res. }\end{array}$ & $\begin{array}{l}\text { A maioria dos profes- } \\
\text { sores classificou sua } \\
\text { qualidade de vida } \\
\text { como "boa", e consta- } \\
\text { tou-se que o perfil } \\
\text { demográfico e profis- } \\
\text { sional não exerceu } \\
\text { influência sobre a } \\
\text { qualidade de vida. }\end{array}$ \\
\hline $\begin{array}{l}\text { Feitosa e } \\
\text { Rodri- } \\
\text { gues } \\
(2010) \text {. }\end{array}$ & $\begin{array}{l}\text { Estudo } \\
\text { descritivo- } \\
\text { exploratório. } \\
\text { Número de } \\
\text { participantes }\end{array}$ & $\begin{array}{l}\text { Os professores } \\
\text { consideraram possuir } \\
\text { saúde e qualidade de } \\
\text { vida satisfatória, mas } \\
\text { buscavam melhorias. }\end{array}$ \\
\hline
\end{tabular}

\begin{tabular}{|c|c|c|}
\hline & $\begin{array}{l}\text { da pesquisa = } \\
72 \text { professo- } \\
\text { res. }\end{array}$ & \\
\hline $\begin{array}{l}\text { Moreira } \\
\text { et al. } \\
(2010 a)\end{array}$ & \begin{tabular}{|l} 
Estudo \\
descritivo \\
correlacional. \\
Número de \\
participantes \\
da pesquisa = \\
654 docentes.
\end{tabular} & $\begin{array}{l}\text { A avaliação interna da } \\
\text { qualidade de vida no } \\
\text { trabalho obteve } \\
\text { índices de correlação } \\
\text { maiores que do estilo } \\
\text { de vida. }\end{array}$ \\
\hline $\begin{array}{l}\text { Moreira } \\
\text { et al. } \\
(2010 b) .\end{array}$ & $\begin{array}{l}\text { Pesquisa } \\
\text { descritiva de } \\
\text { corte trans- } \\
\text { versal. } \\
\text { Número de } \\
\text { participantes } \\
\text { da pesquisa = } \\
654 \text { professo- } \\
\text { res. }\end{array}$ & $\begin{array}{l}\text { Com o avanço na } \\
\text { carreira docente, } \\
\text { aumenta a insatisfação } \\
\text { da qualidade de vida } \\
\text { no trabalho e os } \\
\text { comportamentos } \\
\text { negativos do perfil do } \\
\text { estilo de vida indivi- } \\
\text { dual, cuja maior } \\
\text { preocupação é o nível } \\
\text { de atividade física. }\end{array}$ \\
\hline
\end{tabular}

Fonte: dados da pesquisa.

Os estudos sobre os ciclos de desenvolvimento profissional (SANTINI, 2004; MOREIRA et al., 2010b) auxiliaram na compreensão dos problemas enfrentados na carreira docente, especialmente porque as investigações concentram-se tanto nos aspectos negativos quanto nos aspectos positivos da profissão docente (BOTH, NASCIMENTO; BORGATTO, 2008a). Dentre os aspetos negativos, têm-se as doenças que os docentes estão propensos a contrair, tais como: estresse elevado; SEP ou burnout; hipertensão; diabetes; obesidade; câncer; coronariopatias; além dos vícios do tabagismo e consumo de álcool e drogas. E, ainda obtém risco à sua saúde devido a má alimentação e falta de atividades físicas (BOTH; NASCIMENTO; BORGATTO 2008a; MOREIRA et al., 2010b; NUNEZ, 2009; SANTINI, 2004; SILVA).

Compreender os problemas enfrentados pelos docentes em sua carreira, sobretudo, voltados às condições de saúde e bem-estar, é importante para o docente tanto em sua vida particular, quanto para sua satisfação no trabalho. 


\section{CONDIÇÕES E SATISFAÇÃO NO TRABALHO DO PROFESSOR.}

As condições de trabalho podem ser fatores desencadeadores de patologias encontradas em docentes, especialmente porque elas determinam e intensificam as cargas de trabalho (BOTH, 2008). A desvalorização da educação é um forte motivo de preocupação, pois tem sido caracterizada como a área que mais enfrenta conflitos e desafios diante de uma sociedade em constantes mudanças (GASPARINE et al., 2006).

A presente pesquisa encontrou que dez publicações tratam do nível de satisfação dos professores de educação física, de qualidade de vida/estilo de vida no trabalho, sendo que 08 delas (BOTH; NASCIMENTO; BORGATTO, 2008a; BOTH; NASCIMENTO; BORGATTO, 2008b; FARIAS et al., 2008; FEITOSA; RODRIGUES, 2010; LEMOS, 2007; LEMOS; NASCIMENTO; BORGATTO, 2007; MOREIRA et al., 2010a; SILVA; NUNEZ, 2009) mostraram que, apesar das dificuldades encontradas na profissão, o nível de satisfação aumenta com o passar dos anos trabalhados, enquanto que dois estudos (BOTH, 2008; MOREIRA et al., 2010b) disseram o oposto, ou seja, que com o passar dos anos diminui o nível de satisfação dos professores de educação física com a sua profissão.

Além das doenças enfrentadas pelo trabalho docente, o professor de educação física encontra diversas dificuldades no seu ambiente de trabalho, como por exemplo, falta de material para desenvolvimento de atividades propostas, relacionamentos interpessoais e má remuneração (BOTH, 2008; FARIA et al., 2008; GASPARINE et al., 2006; LEMOS; NASCIMENTO;
BORGATTO, 2007; MOREIRA et al., 2010a; MOREIRA et al., 2010b).

A percepção do indivíduo em relação ao bem-estar pode sofrer influência de fatores socioambientais e de fatores individuais (MOREIRA et al., 2010b). Desse modo, o professor de educação física sofre negativamente com sua condição de trabalho, na qual fatores físicos, fisiológicos, psíquicos e locais para sua atuação repercutem em seu trabalho e em sua qualidade de vida (LEMOS; CRUZ, 2005).

Um estudo realizado por Farias et al. (2008), no qual participaram 380 professores da rede de ensino estadual do Rio Grande do Sul, Brasil, teve o intuito de investigar a qualidade de vida no trabalho, e evidenciou que $66,1 \%$ dos profissionais estavam satisfeitos com a docência, 25,8\% indecisos e $8,2 \%$ insatisfeitos. Observa-se, que os resultados do estudo de Lemos, Nascimento e Borgatto (2007) foram similares, no qual os professores de educação física apresentaram percepções positivas com relação ao seu estilo de vida.

\section{ESTILO DE VIDA E PRÁTICAS QUE MELHORAM O BEM- ESTAR DO TRABALHADOR DO- CENTE}

Bem-estar é um conceito bastante utilizado no âmbito acadêmico, e está relacionado a questões que vão desde aspectos pessoais à aqueles de cunho sociocultural e econômico (FEITOSA; RODRIGUES, 2010). Para os autores Both, Nascimento e Borgatto (2008b), a condição humana é resultante de um conjunto de parâmetros individuais e socioambientais, modificáveis ou não, que caracterizam as condições em que vive o ser humano.
Both, Nascimento e Borgatto (2008b), Feitosa e Rodrigues (2010), relataram que para realizar um trabalho preventivo voltado ao professor de educação física, deve-se compreender a saúde do trabalhador docente considerando os ciclos de desenvolvimento profissional, a satisfação no trabalho e o estilo de vida.

A profissão docente é uma das mais estressantes devido às longas jornadas de trabalho, que começam logo cedo, pela manhã, chegando até a noite. Por conta dessa jornada, o professor é forçado a desrespeitar seus horários habituais, perdendo horas de sono, alimentando-se mal e não usufruindo de momentos de lazer (FEITOSA; RODRIGUES, 2010).

Feitosa e Rodrigues (2010) apontaram que a satisfação no trabalho é uma via de mão dupla, que atende tanto às necessidades do trabalhador com a elevação da satisfação, da motivação, da preservação da sua saúde física e psicológica, quanto para a empresa no que se refere ao seu fortalecimento e aumento da produtividade.

Os autores Both, Nascimento e Borgatto (2008b) evidenciaram que a autonomia no trabalho pedagógico, a possibilidade de progredir na carreira docente, as garantias legais que regem a atuação docente e lhe dão suporte e a percepção da importância da função de ser professor compreendem indicadores que interferiram positivamente na avaliação global da qualidade de vida no trabalho.

Com base nos autores Both, Nascimento e Borgatto (2008b), e em Feitosa e Rodrigues (2010), nota-se que os trabalhos preventivos para qualidade de vida do professor de educação física devem estar relacionados à satisfação do trabalho docente, que pode estar aliado à boa remuneração, condições de trabalho adequado, plano 
de carreira curta, tempo para exercer suas atividades domésticas e de lazer.

\section{CONSIDERAÇÕES FINAIS}

Ao considerar os resultados do presente estudo, verificou-se que o nível de satisfação aumenta com o passar dos anos na profissão, entretanto, encontrou-se que a má alimentação e má remuneração, somadas à carga horária elevada são fatores que contribuem para a diminuição do bem-estar dos professores de educação física, sobretudo, para o aparecimento de doenças relacionadas ao exercício profissional.

Os estudos pesquisados tendem a observar à importância da contribuição do estilo de vida e da qualidade de vida para o trabalhador docente, os quais estão diretamente relacionados ao bem-estar.

A característica metodológica dos estudos avaliados foi quantitativa, o que mostra a importância de incentivar-se mais estudos qualitativos para inserir novos olhares ao analisar o bem-estar de professores de educação física.

Os estudos convergiram no sentido de que para melhorar o bem-estar dos professores de educação física, deve haver um investimento governamental na educação, que proporcione um período mais curto no plano de carreira docente, uma boa remuneração, espaços e materiais adequados.

\section{REFERÊNCIAS}

BOTH, Jorge. Qualidade de vida na carreira docente em educação física do magistério público estadual de Santa Catarina. 2008. 120f. Dissertação (Mestre em Educação Física)Universidade Federal de Santa Catarina, área de concentração, teoria e prática pedagógica em Educação Física, programa de pós- graduação em Edu- cação Física. Florianópolis, 2008.

Disponível em:

https://repositorio.ufsc.br/handle/1234 $56789 / 91795$

BOTH, Jorge. Bem-estar do trabalhador docente em educação física da região sul do Brasil. 2011. $248 f$. Dissertação (Doutorado em Educação Física)- Universidade Federal de Santa Catarina, área de concentração, teoria e prática pedagógica em Educação Física, programa de pós- graduação em Educação Física. Florianópolis, 2011. Disponível em:

https://repositorio.ufsc.br/handle/1234 $56789 / 95232$

BOTH, Jorge; NASCIMENTO, Juarez Vieira do; BORGATTO, Adriano Ferreti. Percepção da qualidade de vida no trabalho ao longo da carreira docente em Educação Física. Revista Brasileira de Cineantropometria \& Desempenho Humano, Santa Catarina, v.10, n.4, p.372-378, 2008a. Disponível em:

<https://periodicos.ufsc.br/index.php/r bcdh/article/viewFile/1980-

0037.2008v10n4p372/5866>. Acesso em: 12 set. 2012.

BOTH, Jorge; NASCIMENTO, Juarez Vieira do; BORGATTO, Adriano Ferreti. Percepção da qualidade de vida no trabalho e perfil do estilo de vida dos docentes de educação física do estado de Santa Catarina. Revista da Educação Física/UEM, Maringá, v. 19, n. 3, p. 377-389, 2008b. Disponível em:

<http://www.periodicos.uem.br/ojs/ind ex.php/RevEducFis/article/view/5993> . Acesso em: 10 set. 2012.

ESTEVE, José. Manoel. Mal-estar docente: a sala de aula e a saúde dos professores. São Paulo: Edusc, 1999.

FARIAS, Gelcemar Oliveira, et al. Carreira docente em educação física: uma abordagem sobre a qualidade de vida no trabalho de professores da rede estadual de ensino do Rio Grande do Sul. Revista da Educação Física/UEM, Maringá, v.19, n.1, p.11-22, 2008.

Disponível em: <http://www.periodicos.uem.br/ojs/ind ex.php/RevEducFis/article/view/4310> . Acesso em: 01 set. 2012.

FEITOSA, Lisarda da Silva Costa; RODRIGUES, Ana Maria da Silva. Saúde e qualidade de vida na percepção dos professores de educação física das escolas estaduais da zona sul de Teresina (PI). In: VI Encontro de Pesquisa em Educação

PPGED/CCE/UFPI, 2010, Teresina (PI). Anais do VI Encontro de Pesquisa em Educação. Teresina, v.00, p.0113, 2010. Disponível em:

$<$ http://leg.ufpi.br/subsiteFiles/ppged/a rqui-

vos/files/VI.encontro.2010/GT.16/GT 16_06_2010.pdf>. Acesso em: 05 out. 2012.

GASPARINI, Cristiane et al. A realidade dos professores de educação física na escola: suas dificuldades e sugestões. Revista Mineira de Educação Física, Viçosa, v.14, n.1, p.109137, 2006.

GIL, Antonio Carlos. Métodos e Técnicas de Pesquisa Social. $5^{\mathrm{a}}$ ed. São Paulo: Atlas, 2006.

LEMOS, Carlos Augusto Fogliarini; CRUZ, Roberto Moraes. Condições e cargas de trabalho da atividade docente. Revista Plural, Florianópolis, p.2027, jun., 2005.

LEMOS, Carlos Augusto Fogliarini. Qualidade de vida na carreira profissional de professores de educação física do magistério público estadual/RS. 2011. 144f. Dissertação (Mestre em Educação Física)- Universidade Federal de Santa Catarina, programa de pós- graduação em Educação Física. Santa Catarina, 2007. Disponível em:

$<$ https://repositorio.ufsc.br/handle/123 456789/89948>. Acesso em: 11 set. 2012.

LEMOS, Carlos Augusto Fogliarini; NASCIMENTO, Juarez Vieira do; BORGATTO, Adriano Ferreti. Parâmetros individuais e sócio-ambientais da qualidade de vida percebida na 
carreira docente em educação física. Revista Brasileira de Educação Física e Esporte, São Paulo, v.21, n.2, p.8193, 2007. Disponível em: <http://www.revistas.usp.br/rbefe/artic le/view/16646>. Acesso em: 12 set. 2012.

MELEIRO, Alexandrina Maria Augusta da Silva. O stress do professor. $7^{\mathrm{a}} \mathrm{ed}$. In: LIPP, Marilda (Org.). O stress do professor. Campinas: Papirus, 2002, p.11-27.

MOREIRA, Hudson de Resende et al. Qualidade de vida do trabalhador docente em Educação Física do estado do Paraná, Brasil. Revista Brasileira de Cineantropometria e Desempenho Humano, Paraná, v.12, n.6, p.435-442, 2010. Disponível em:

<http://www.scielo.br/pdf/rbcdh/v12n6 $/ v 12$ n6a07.pdf $>$. Acesso em: 05 out. 2012.

MOREIRA, Hudson de Resende et al. Qualidade de vida no trabalho e perfil do estilo de vida individual de professores de Educação Física ao longo da carreira docente. Revista Motriz, Rio Claro, v.16, n.4, p.900-912, 2010.

Disponível em:

<http://www.scielo.br/pdf/motriz/v16n 4/a10v16n4.pdf $>$. Acesso em: 10 out. 2012.

REIS, Eduardo José Farias Borges dos et al. Trabalho e distúrbios psíquicos em professores da rede municipal de Vitória da Conquista. Bahia, Brasil. Cadernos de Saúde Pública, Rio de Janeiro, v.21, n.5, p.1480-1490, set/out., 2005. Disponível em: <http://www.scielo.br/pdf/\%0D/csp/v2 1n5/21.pdf >. Acesso em: 01 set. 2012.

\section{SANTINI, Juarez. A síndrome de} esgotamento profissional: o "abandono" da carreira docente pelos professores de educação física da rede municipal de ensino de porto alegre. 2004. 224f. Dissertação (Mestrado em Educação Física)- Universidade Federal do Rio Grande do Sul, programa de pós- graduação em ciência do movimento humano. Porto Alegre, 2004. p.224. Disponível em: http://www.lume.ufrgs.br/handle/1018 3/4299

SILVA, Junior Vagner Pereira da; NUNEZ, Paulo Ricardo Martins. Qualidade de vida, perfil demográfico e profissional de professores de educação física. Revista Pensar a Prática, Campo Grande, v.12, n.2, p.1-11, mai/ago., 2009. Disponível em: $<$ https://www.revistas.ufg.br/fef/article /view/3795>. Acesso em: 12 set. 2012.

TEODORO, António. Da profissionalização da actividade docente à crise de identidade dos professores: considerações preliminares para um estudo da situação portuguesa. Boletim da Sociedade Portuguesa de Educação Física, Lisboa, n.9, p.37-54, 1994. Disponível em:

<https://boletim.spef.pt/index.php/spef /article/view/77>. Acesso em: 01 set. 2012. 